\title{
Spinal dysraphism
}

\author{
D. N. Grant \\ F.R.C.S. \\ The Hospital for Sick Children, Great Ormond Street, London, W.C.1, and \\ The National Hospital, Queen Square
}

\section{Introduction}

Spinal dysraphism is a term which was revived by Lichtenstein (1940) under which he grouped disorders arising from cutaneous, mesodermal or neural derivatives of the dorsal median region of the developing embryo. Within this broad classification must be included the sub-groups of spina bifida aperta with myeloschisis, myelomeningocele or meningocele and spina bifida occulta with or without associated neuroectodermal and mesodermal abnormalities. Although grouping these disorders under the single heading of spinal dysraphism need not imply uniformity of aetiology or pathogenesis, this is of course considered by many to be the case (Brocklehurst, 1971). There is a group of disorders of development of the dorsal mid-line of the embryo including involvement of ectodermal, mesodermal, neural or endodermal elements which is in some respects separate from the various degrees of spina bifida cystica. It is this particular group, which includes such apparently diverse conditions as diastematomyelia, diplomyelia, dermoid cyst or sinus, lipoma of the conus medullaris, prolongation of the conus and a variety of fibrous bands between conus, nerve roots and dura, that I propose to consider. There may be slight theoretical justification for regarding this as an independent group, but, for practical purposes, it is useful to do so, because the conditions are so different from the commoner spina bifida cystica in their presentation, investigation, treatment and prognosis (Till, 1969).

\section{Presentation}

This may occur at any age from birth to adulthood. Clearly babies which have dorsal cutaneous stigmata, sufficiently gross to be considered significant by parent or family doctor, present early. The possible lesions seen on the skin, usually in the lumbar or sacral regions, include patches of hair which may be sufficiently profuse to merit the term 'faun's tail' or may simply be a slight exaggeration of the normal cutaneous down; cutaneous naevi and dermal scars or sinuses. The sinus opening may be in or close to the mid-line, commonly is sacral in situation but may be at any level. It is to be dis- tinguished from the pilo-nidal sinus. Subcutaneous lesions which are visible or palpable through the skin also tend to cause early presentation. These include subcutaneous lipomata and dermoid cysts. Palpable through the skin may be bony abnormalities sufficiently obvious to be noted by the parents. At an early age, differences in the dimensions of the feet or legs, or abnormality of the posture of the foot or leg on one side, may be noted. Such differences, unless gross, are more likely to present when the child begins to walk. At this stage, and during the following years, many children present to orthopaedic surgeons because of asymmetry of the legs or feet, abnormalities of gait or scoliosis.

Difficulty in achieving control of micturition may be the mode of presentation and this may naturally be at a later date than difficulty in walking. The presence of bony abnormalities usually more extensive than simple spina bifida of one segment, detected during coincidental radiography of the trunk, may result in the child's referral for further investigation. Presentation may be delayed for many years, even into adult life, until the onset of progressive abnormalities of the lower limbs or gait. Rarely meningitis may draw one's attention to the presence of a dermal sinus. Such a sinus may be exceedingly small but should be sought particularly in children with recurrent attacks of meningitis or if the responsible organism is an unusual one.

\section{Physical examination}

The cutaneous stigmata already mentioned may be obvious. Dermal sinuses may be a source of difficulty. A postanal dimple is a relatively common finding and in the absence of other abnormalities detected either clinically or by X-rays is unlikely to be significant. Subcutaneously may be a palpable lipoma which is sometimes little more than a pad of fat. The underlying bony abnormality may be detectable as scoliosis, irregularity or absence of spinous processes. The lower limbs may be clearly asymmetrical in their development, the abnormal side being less in length, girth or both. In addition to shortening and narrowing there may also be various degrees of pes cavus or of talipes. Power is not 
usually diminished when the condition is first detected. There may occasionally be sensory impairment of sufficient severity to result in trophic ulceration of the toes. The tendon reflexes may be absent or diminished on the affected side but this is by no means invariable. The plantar response too, may be absent. When standing, the inequality of the legs may result in a compensatory scoliosis although this may equally be due to bony spinal abnormality. When walking, there may be a limp, with a tendency to inversion and adduction of the affected foot.

\section{Investigation}

Under any of the circumstances mentioned, spinal radiographs should be obtained. Noabnormality may be seen but this is exceptional in those patients in whom a dysraphic lesion is subsequently demonstrated. A simple degree of spina bifida with mid-line cleft is also unlikely to be the only bony accompaniment of a significant underlying abnormality. Complex spina bifida with asymmetrical clefts involving several segments is more commonly of significance. There may be clear evidence of diastematomyelia, when the bony spur splitting the spinal cord is visible. This is commonly associated with localized widening of the spinal canal and a narrow disc space. Widening of the canal without evidence of a spur may indicate an intraspinal lipoma or dermoid. If there is a wide defect in the bone together with a subcutaneous mass, then it is likely that the intraspinal lesion, be it lipoma or dermoid, is continuous with the subcutaneous lesion. Anterior defects include hemivertebrae, fusion of adjacent bodies and sagittal splitting which may be suggestive of a neurenteric cyst.

If it is decided to carry investigations further, the procedure of choice is considered to be cisternal air myelography. Air is preferable to positive contrast media for two principal reasons. Dense media obscure the presence of a fibrous band or prolonged conus which may be responsible for tethering of the lower end of the spinal cord. Air, on the other hand, satisfactorily outlines the conus and any abnormal prolongation. The other advantage is that the air is completely re-absorbed and the investigation may be repeated without the confusion caused by residual positive contrast media. The small risk of causing air embolism may be completely avoided by being satisfied only by a perfect cisternal puncture and by varying the quantity and pressure of the injected air according to the estimated capacity of the spinal subarachnoid space. The cisternal route is chosen because there is a risk of lumbar puncture either damaging the lesion under investigation or being unsuccessful because the subarachnoid space cannot be found. The objection may be raised that if an Arnold-Chiari malformation is present, there is a risk of damaging the abnormally situated cerebellar tonsils. In practice, in the course of many such investigations, the Arnold-Chiari malformation has not been found to be associated with this sub-group of spinal dysraphism. Air myelography allows one to see the extent of the subarachnoid space, the level at which the conus of the spinal cord ends and whether it shows the normal degree of mobility. This is determined by taking radiographs prone and supine. The conus should normally lie at the level of the disc space between first and second lumbar vertebrae. Swelling of the conus indicative of lipoma or dermoid can readily be seen. Diastematomyelia can be very convincingly demonstrated when air lodges in the cleft between the two half-cords. Tomography may be used if there is difficulty in interpreting the ordinary myelographic views. In adolescents or adults tomography is usually necessary because of the density of the overlying bony shadows.

At this point it would be as well to indicate the rationale for undertaking investigations in patients suspected to be suffering from dysraphism. This entails a brief consideration of the mechanism of production of damage in the lower end of the spinal cord. It is unlikely that interference with 'ascent of the conus' is a major factor in producing neurological damage. Such ascent, resulting from differential growth of the bony canal and the nervous tissue, is very slight, the conus rising from third lumbar to second lumbar levels between the twenty-sixth week of pregnancy and maturity. Of more significance is the fact that in many dysraphic children, the portion of the developing spinal cord caudal to the neuropore, which usually atrophies to produce the filum terminale, fails to do so. The result is a prolonged conus of substantial girth. This prevents the normal mobility of the lower end of the spinal cord, and it is postulated that movements of the trunk such as flexion and extension, by transmitting tension through the prolonged conus to the spinal cord, result in neuronal damage. There is also the probability that whatever causes the obvious lesions of ectoderm and mesoderm, also causes a lesion of the developing nervous elements. Clearly, in dealing with the former mechanism, if one can restore mobility to the conus, one might prevent progressive damage, whereas, with intrinsic damage to developing nervous tissue, one is unable in our present state of knowledge to influence the damage once it has occurred. It is in the light of these postulated pathogenetic mechanisms that a rationale for investigation and treatment has evolved. It has to be accepted that neurological damage, unless it has resulted from recent interference with the cord, is irreversible and that associated developmental inequalities of the extremities will not be reversed. One's effort is directed towards preventing the onset of neurological damage or of 
arresting a situation in which progressive damage is occurring.

The decision to investigate by myelography is easy in a child undergoing progressive deterioration but it is not so easy in a child who is neurologically intact. However, if one accepts the basic postulates, one is driven to earlier investigation and treatment in order to provide the best prophylaxis. If a patient presents with any of the skin lesions mentioned, together with evidence of underlying bony abnormality, then myelography is indicated irrespective of the state of the lower extremities. Although it is unlikely that such a skin lesion will co-exist with a dysraphic lesion of the cord without overlying bony abnormality, it is nevertheless possible, and bony normality should not preclude myelography, although making it much less likely to be positive. Air myelography may be proceeded with at the age of about 6 weeks onwards.

\section{Treatment}

After myelography the rationale for proceeding to operation follows similar lines of argument. If no abnormality of anatomy of the cord or of its mobility is demonstrated then there is no indication for operation. There are occasional exceptions to this rule. A dermal sinus requires exploration even if the cord appears normal on the myelogram. If there is evidence of progressive neurological or developmental abnormality together with an abnormal myelogram demonstrating tethering of the spinal cord or compression by any of the lesions mentioned, operation, in an attempt to relieve the tethering or pressure, is indicated. The decision is more controversial when considering a patient who has either no clinically demonstrable developmental or neurological abnormality or in whom such abnormality has remained static for a long period. I believe that operation should be recommended in infancy or childhood in an attempt to forestall the deterioration which undoubtedly can occur in a proportion of such patients.

If the patient is first seen in adolescence or adult life with a static neurological abnormality it would appear to be reasonable to review the patient's condition at regular intervals believing that the longer the onset of complications is delayed the less likely they are to appear. In such a patient early detection of deterioration would hold out an excellent prospect of arresting the process by operation.

The operation in its many variations consists of an attempt to return mobility to the spinal cord by division of tethering bands or adhesions, removal of bony spurs or excision of lipomata and dermoid cysts. If there is technical difficulty in obtaining a complete freeing of the spinal cord I would always try to err on the side of being conservative rather than causing neurological damage. This applies particularly to attempted excision of a spinal cord lipoma which may be intimately related to functioning nervous tissue. Many such lesions have been partially excised with satisfactory long-term results. Fortunately the operation is rarely followed by complications. The commonest is leakage of CSF from the wound due to incomplete closure of the dura. This usually responds to conservative measures which must include antibiotics to prevent meningitis. Much less common is aggravation of the neurological abnormality by trauma at the time of the operation. With very few exceptions this has proved to be temporary.

While the evidence that progressive neurological abnormalities associated with dysraphism can be arrested by operation is undoubted, there is as yet no conclusive proof that early operation does forestall the onset of such abnormalities. This evidence could be obtained by a prospective controlled trial but to embark on such a trial would hardly be ethical when one believes that the benefits of operation greatly outweigh the risks.

\section{References}

Brocklehurst, G. (1971) The pathogenesis of spina bifida. Developmental Medicine and Child Neurology, 13, 147.

Lichtenstein, B.W. (1940) Spinal dysraphism. Archives of Neurology and Psychiatry, 44, 792.

Till, K. (1969) Spinal dysraphism. Journal of Bone and Joint Surgery, 51B, 415. 